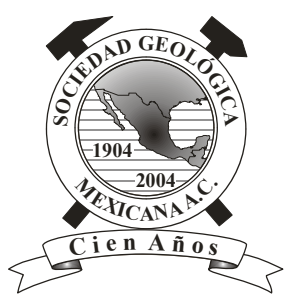

\title{
Potencial energético del Alto Golfo de California
}

\author{
Gerardo Hiriart Le Bert ${ }^{1, *}$ \\ ${ }^{1}$ Proyecto IMPULSA IV, Instituto de Ingeniería, \\ Universidad Nacional Autónoma de México, Ciudad Universitaria, \\ Delegación Coyoacán, 04510 México D.F., México
}

\begin{abstract}
Resumen
El alto Golfo de California, además de riqueza ecológica, pesquera y minera, tiene un gran potencial energético. En este trabajo se presenta una evaluación preliminar de lo que idealmente se podría obtener en energía eléctrica si se aprovecharan estas fuentes renovables. Se examina el potencial, en potencia y energía, que se podría producir a partir de; (a) cambios de nivel de las mareas, (b) corrientes de marea, (c) manifestaciones geotérmicas costeras, (d) el gradiente salino en la desembocadura del Río Colorado, y (e) las emisiones hidrotermales submarinas. Las conclusiones son sorprendentes en cuanto al enorme potencial energético que hay en las mareas y en las emisiones submarinas. Se hace hincapié en que esta evaluación del potencial energético no considera por el momento los impactos ambientales ni los métodos constructivos, ya que éstos se estudiarán más adelante.
\end{abstract}

Palabras clave: Energías renovables, corrientes marinas, energía de las mareas, ventilas hidrotermales, geotermia

Abstract

The upper part of the Gulf of Baja California, besides the ecological diversity and mineral resources, has a great potential in energy resources. In this paper it is presented a preliminary evaluation of the ideal actions in order to obtain electric energy if renewable resources were used.

The potential in power and energy that could be produced is evaluated from: (a) tide level changes, (b) tide currents, (c) coastal geothermal manifestations, (d) saline gradients in the discharge area of the Colorado River, and (e) submarine hydrothermal vents. It is emphasized that this evaluation assesses the energetic potential only and does not consider the environmental impact and the constructive methods, as these issues are being studied separately.

\section{Introducción}

Además del alto valor ecológico y de biodiversidad que posee el extremo norte del Golfo de California, también existen importantes recursos de energías renovables que conviene examinar.

La enorme amplitud de las mareas (hasta $6 \mathrm{~m}$; CICESE, 2006), que se observan desde Puerto Peñasco y San Felipe hasta el norte del Golfo de California, está causada por un fenómeno de resonancia hidráulica, poco frecuente en la naturaleza. En éste la marea que se genera a la entrada del Golfo, frente a Mazatlán y Los Cabos, viaja a una velocidad tal que el tiempo que le toma en alcanzar la cabecera del Golfo y regresar coincide con el periodo de subida y bajada de la marea. Esto provoca que una marea de $1.2 \mathrm{~m}$ en Mazatlán se amplifique a más de $6 \mathrm{~m}$ en el extremo norte del Golfo de California.

Por otro lado, la entrada y salida de estas enormes masas de agua provoca fuertes corrientes marinas en las zonas más estrechas del Golfo, tales como el Canal del Infiernillo frente a la Isla Tiburón, el Canal Ballenas frente a la Isla Ángel de la Guarda, y el estrecho entre dichas islas. 
La separación de la Península de Baja California respecto a la Placa de Norteamérica, iniciada en el Mioceno (Martín-Barajas, 2000) y que actualmente tiene lugar a una velocidad promedio de $5 \mathrm{~mm}$ por año (Hole et al., 2007), es la causa del vulcanismo reciente y de los sistemas de fallas normales y transcurrentes que favorecen el transporte de calor hasta la superficie. Ello ocasiona numerosos manantiales hidrotermales someros a lo largo de la costa de la península (Vidal et al., 1978; Prol-Ledesma et al., 2004; Canet y Prol-Ledesma, 2007; Camprubí et al., 2008) y en el fondo del Golfo.

Finalmente, las grandes planicies del Delta del Colorado están expuestas a una de las radiaciones solares más intensas del mundo (Almanza et al., 2003). Ello ocasiona que la salinidad de las aguas que entran y salen, cubriendo con una delgada lámina los suelos arenosos, cambie en pocas horas. Esto ocurre frente a la descarga de agua dulce del Río Colorado.

Todos los fenómenos anteriormente descritos encierran un enorme potencial energético que eventualmente se podría aprovechar para la generación de energía eléctrica. En el marco del Programa IMPULSA IV de la UNAM "Desalación de agua de mar con Energías Renovables" (Alcocer y Hiriart, 2008) se ha realizado una evaluación preliminar de este potencial, sin entrar todavía al estudio de su viabilidad económica y su eventual impacto ambiental. Así, la estimación presentada en este trabajo fue realizada solamente con el fin de evaluar el potencial de cada uno de los fenómenos analizados.

\section{Energía de las mareas}

Un ascenso del nivel del agua de $6 \mathrm{~m}$ en 6 horas, como el que tiene lugar en el extremo norte del Golfo de California, equivale a un trabajo de $50 \mathrm{MWh} / \mathrm{Km}^{2}$ y a una potencia media de $15 \mathrm{MW} / \mathrm{km}^{2}$. Si se construyera un dique desde la Península de Baja California hasta el continente, con las compuertas adecuadas para dejar que en la parte alta se embalsara agua al subir la marea y luego se cerraran al momento de bajar (obligando al embalse a vaciarse a través de una gran batería de turbinas hidráulicas), la potencia eléctrica que teóricamente se podría generar es de varios miles de MW. En la Fig. 1 se presentan esquemáticamente distintas configuraciones posibles del dique y se calcula, con valores ideales de eficiencia, la potencia y energía de cada arreglo.

Los estudios realizados por el grupo de trabajo integrado mediante el Programa IMPULSA IV han permitido extender este análisis a lugares más restringidos de configuración adecuada, donde se podrían manejar simultáneamente dos embalses menores, uno de alto nivel y otro de bajo nivel. Ello permitiría la generación continua entre el estanque alto y el estanque bajo. En la Fig. 2 se presenta un esquema del funcionamiento de una mareomotriz de doble embalse. Sin tomar en cuenta todavía los aspectos ambientales, se han identificado lagunas que podrían producir más de 50 MW por el método del doble embalse.
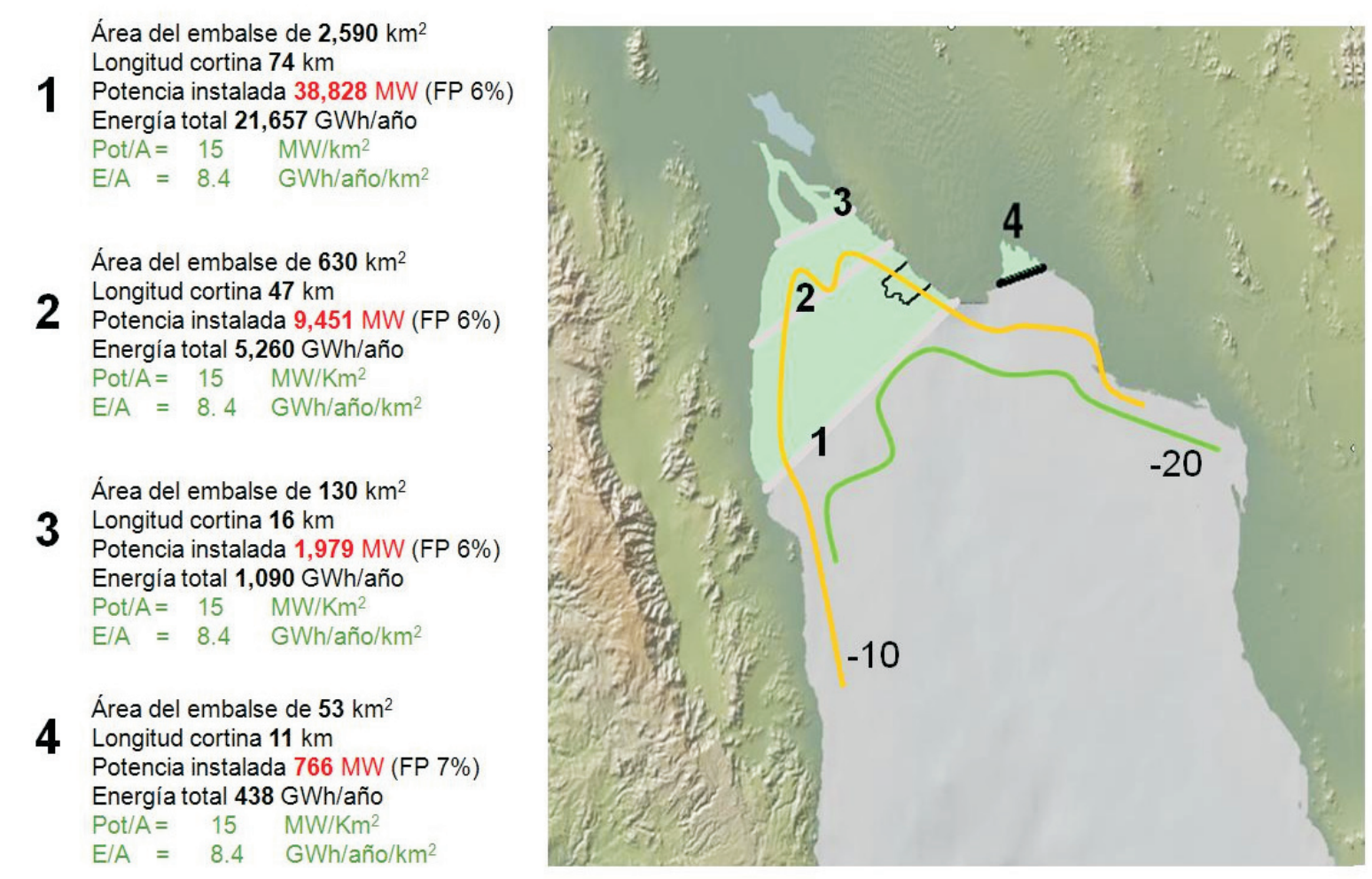

Figura 1. Esquemas teóricos para el aprovechamiento de mareas (contornos de batimetría, en m). 


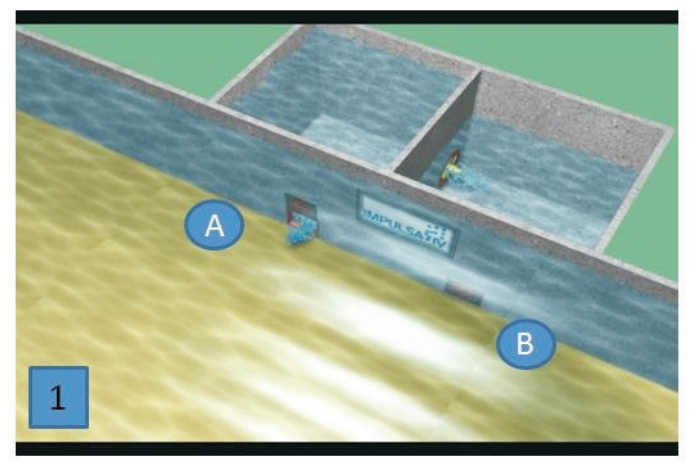

1. Cuando hay marea alta se abre la compuerta delembalse A para mantenerlo lleno.

2. Con marea baja se abre la compuerta B y se mantiene el nivel lo más bajo posible.

3. Se aprovecha el desnivel de ambos embalses para generar con turbinas hidráulicas.
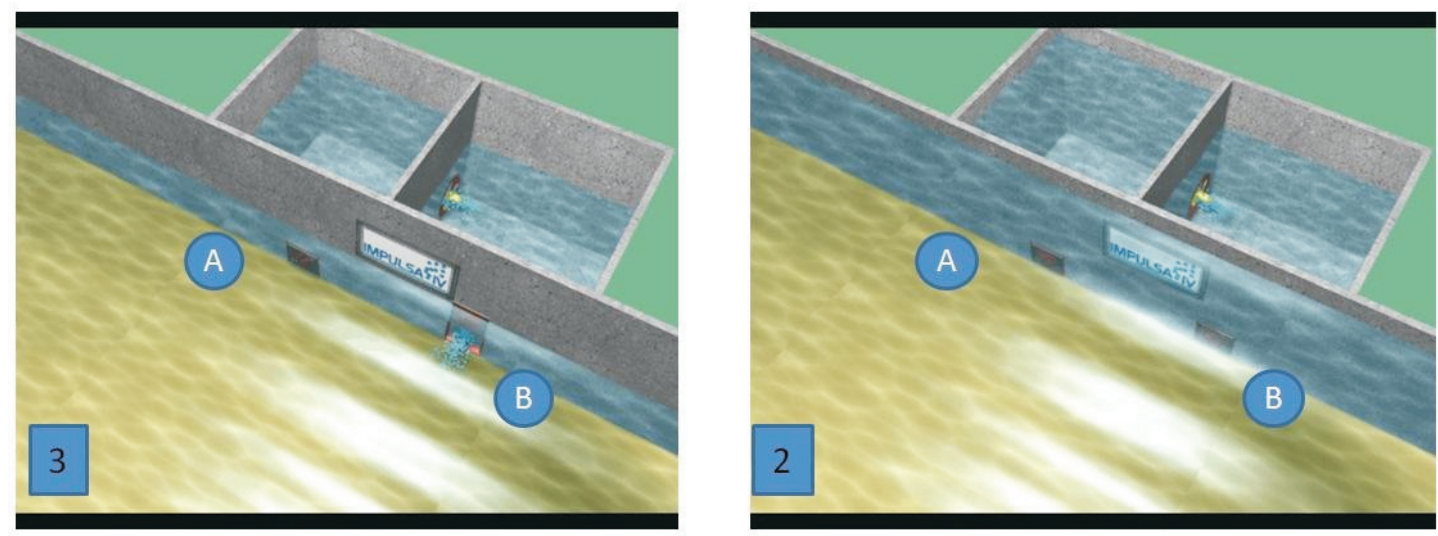

Figura 2. Esquema de generación eléctrica usando mareas con doble embalse.

\section{Corrientes marinas}

Las fuertes corrientes que induce la marea frente a las islas Tiburón y Ángel de la Guarda implican, durante las horas punta, una potencia aprovechable de $5 \mathrm{~kW} / \mathrm{m}^{2}$. Es decir, analizando la situación en forma teórica, se podrían obtener cientos de MW con dispositivos de generación de corrientes marinas en los canales. Estos dispositivos pueden ser turbinas ancladas, turbinas flotantes o una lancha con generadores hidráulicos verticales instalados en la parte inferior de ésta.

\section{Geotermia costera}

A lo largo de toda la Península de Baja California se observan pozos de agua con temperaturas anormalmente altas. La actividad volcánica y tectónica de la zona origina las anomalías geotérmicas que se pueden detectar en algunas playas. Tal es el caso de Los Cabos, en una zona cercana a la planta desaladora local, donde se midieron $84^{\circ} \mathrm{C}$ en un pozo playero y, más notable aún, la zona de $\mathrm{La}$ Joya en Punta Banda, cerca de Ensenada, donde al retirarse la marea se han podido medir hasta $96^{\circ} \mathrm{C}$ en la superficie mojada de la arena (Gutiérrez y Espíndola, 2008). Este potencial es aprovechable para la generación de energía eléctrica mediante pequeñas plantas de ciclo binario. Se estima que en toda la península se podrían generar más de $50 \mathrm{MW}$ aprovechando las anomalías geotérmicas con tecnología binaria y pozos de pequeña profundidad.

\section{Ventilas submarinas}

Ligadas a la extensión tectónica que originó corteza oceánica en el Golfo de California durante los últimos 3-4 millones de años (Martín-Barajas, 2000), se han identificado sistemas hidrotermales submarinos con temperaturas de hasta $300^{\circ} \mathrm{C}$. La presencia de estas ventilas es notoria en la Cuenca de Guaymas frente al puerto de este nombre (p.e., Von Damm et al., 1985), y en las cuencas de Wagner y Consag frente a Puerto Peñasco (Canet et al., 2009). En el Programa IMPULSA IV se ha diseñado un equipo de generación (Hiriart y Espíndola, 2007), que consiste en una planta de ciclo binario, encapsulada en un tubo hermético que tiene en un extremo un intercambiador de calor equivalente a la caldera del circuito y, en el otro, un enfriador que sirve para condensar el vapor del ciclo con el agua fría del mar. Si se lograra bajar cuidadosamente este equipo con el intercambiador-caldera colocado exactamente sobre la ventila submarina se lograrían las condiciones adecuadas para generar electricidad. Se calcula que con una sola ventila de $300^{\circ} \mathrm{C}$ se podrían generar aproximadamente hasta $20 \mathrm{MW}$ eléctricos. Tanto el diseño del equipo de generación como la cuantificación y caracterización de las ventilas están siendo realizados en el marco del Programa IMPULSA IV para lograr eventualmente un aprovechamiento sustentable y económico de esta fuente de energía renovable tan poco conocida en la actualidad (Hernández, 2008). De resultar ciertas las estimaciones preliminares, ésta constituiría una 
fuente de varios cientos de MW a lo largo del Golfo de California.

\section{Gradientes salinos}

Los gradientes salinos extremadamente altos que se producen en la desembocadura del Río Colorado (cuando éste lleva agua, lo que ocurre de forma intermitente) pueden ser utilizados para elevar agua de mar a algún alto topográfico, utilizando la presión osmótica que se produce al separar dos fluidos con una membrana semi-impermeable. Esta tecnología se está estudiando en Noruega y parece ser atractiva cuando se usa agua de mar y dulce en la descarga de algún río; en el caso de la desembocadura del Río Colorado el agua de mar posee el doble de la salinidad normal (Cath et al., 2006), por lo que la presión osmótica es considerablemente mayor que en el caso de la salinidad normal.

El gran potencial en energías renovables de la parte alta del Golfo de California hace necesario continuar estos estudios, con el fin de afinar su evaluación teniendo en cuenta los aspectos ambientales, sociales y económicos.

\section{Agradecimientos}

Agradezco al Ing. L.C.A. Gutiérrez Negrin sus valiosos comentarios. Esta investigación se está realizando en el marco del Programa IMPULSA IV (www.impulsa4.unam.mx).

\section{Referencias bibliográficas}

Alcocer, S.M., Hiriart, G., 2008, An Applied Research Program on Water Desalination with Renewable Energies: American Journal of Environmental Science, 3, 204-211.

Almanza, R., Callejas, O., Correa, G., 2003, Ingeniería de la Energía Solar II: Universidad Nacional Autónoma de México, Instituto de Ingeniería, ISBN 970-32-1153-4.

Camprubí, C., Canet C., Rodríguez-Díaz, A.A., Ledesma Prol., R.M., Blanco-Florido, D., Villanueva, R.E., López-Sánchez, A., 2008, Gelogy, ore deposits, and hydrothermal venting in Bahía Concepción, Baja California Sur, México: The Island Arc, 17, 6-25.

Cath, T.Y., Chidress, A.E., Elimelech, M., 2006, Forward osmosis: Principles, Applications, and Recent Developments: Journal of Membrane Science, 281, 70-87.

Canet, C., Prol-Ledesma, R.M. (2007): Mineralizing processes at shallow submarine hydrothermal vents. En: Alaniz-Álvarez, S.A., Nieto-Samaniego, A.F. (Eds.), Geology of México: Celebrating the Centenary of the Geological Society of México. Geological Society of America Special Paper 422, 359-376.

Canet, C., Prol-Ledesma, R.M., Dando, P.R., Vázquez-Figueroa, V., Birosta, E., Villanueva-Estrada, R.E., Camprubí, A., Robinson, C.J., Shumilin, E., Franco, S.I., Peláez-Gaviria, J.R., Estradas, A., Hiriart, G., Sánchez, A., Rodríguez-Figueroa, G., Tauler, E.: Discovery of massive gas seepage along the Wagner Fault, Northern Gulf of California. Marine Geology (aceptado).

Camprubí, A., Robinson, C.J., Shumilin, E., Franco, S.I., Peláez-Gaviria, J.R., Estradas, A., Sánchez, A., Hiriart, G., Rodríguez-Figueroa, G., Tauler, E., 2009, Discovery of massive gas seepage along the Wagner Fault, Northern Gulf of California: Marine Geology (enviado). Centro de Investigación Científica y de Educación Superior de Ensenada, Baja California (CICESE), 2006. www.cicese.edu.mx

Gutiérrez, H., Espíndola, S., 2008, Using coastline geothermal resources for desalination in Baja California, Mexico (resumen), en 2nd International Conference on Ocean Energy (ICOE 2008): Brest, Francia.

Hiriart, G., Espindola, S., 2007, Aprovechamiento de las ventilas hidrotermales para generar electricidad (resumen), en VIII Conf. Anual de la AMEE, 153-159.

Hernández, I., 2008, Factibilidad técnica de la generación eléctrica con ventilas hidrotermales. Instituto de Ingeniería, UNAM, Tesis de Licenciatura.

Hole, W., Lizarralde, D., Gary, J., 2007, Variation in styles of rifting in the Gulf of California: Nature, 448, 466-469.

Prol-Ledesma, R.M., Canet, C., Torres-Vera, M.A., Forrest, M.J., Armienta, M.A., 2004, Vent fluid chemistry in Bahía Concepción coastal submarine hydrothermal system, Baja California Sur, México: Journal Volcanology and Geothermal Research, 137, 311-328.

Martín-Barajas, A., 2000, Volcanismo y extensión en la Provincia Extensional del Golfo de California: Boletín de la Sociedad Geológica Mexicana, 53, 72-83.

Vidal, V.M.V., Vidal, F.V., Isaacs, J.D., 1978, Coastal submarine hydrothermal activity off Northern Baja California: Journal of Geophysical Research, 83-B, 1757-1774.

Von Damm, K.L., Edmond, J.M., Measures, C.I., Grant, B., 1985. Chemistry of submarine hydrothermal solutions at Guaymas Basin, Gulf of California. Geochemical et Cosmochimica Acta, 49, 2221- 2237. 